International Journal of Pure and Applied Mathematics

Volume 87 No. $6 \quad 2013,845-853$

ISSN: 1311-8080 (printed version); ISSN: 1314-3395 (on-line version)

url: http://www.ijpam.eu

doi: http://dx.doi.org/10.12732/ijpam.v87i6.13

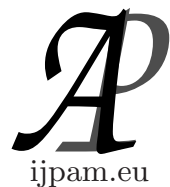

\title{
ROUGH MATRIX THEORY AND ITS DECISION MAKING
}

\author{
S. Vijayabalaji ${ }^{1}$, P. Balaji ${ }^{2}$ \\ ${ }^{1}$ Department Mathematics \\ University College of Engineering Panruti \\ A Constituent College of Anna University Chennai \\ Panruti, 607106, Tamilnadu, INDIA \\ ${ }^{2}$ Department of Mathematics \\ R.M.D. Engineering College
}

Kavaraipettai, 601 206, Tamilnadu, INDIA

\begin{abstract}
Rough set theory was introduced by Pawlak in the early 1980's to deal with a vague description of objects. Rough sets allow for representing sets approximately in terms of the available context knowledge. The concept of approximation is one of the most fundamental in rough set theory. The purpose of this paper is to introduce the new structure called Rough Matrix Theory,we also making the basic notions in our new way of representations. Using this rough matrix we deal with a decision making theory.
\end{abstract}

AMS Subject Classification: 94D05, 68T37

Key Words: rough set, rough membership function, rough approximations, rough matrix

\section{Introduction}

Rough set theory, introduced by Pawlak [3] expresses vagueness, not by means of membership, but employing a boundary region of a set. If the boundary region of a set is empty it means that the set is Crisp, otherwise the set is rough (inexact).

Received: September 6, 2013

(c) 2013 Academic Publications, Ltd. url: www.acadpubl.eu 
Rough set theory can be regarded as a new mathematical tool for imperfect data analysis. The theory has found applications in many domains, such as decision support, engineering, environment, banking, medicine and others. Rough set concept can be defined by means of topological operations, interior and closure, called approximations.

Pawlak [3] stated that, Suppose we are given a set of objects $U$, called the universe and an indiscernibility relation $R \subseteq U \times U$, representing our lack of knowledge about elements of $U$. Hence Pawlak assumed that $R$ is an equivalence relation. Let $X$ be a subset of $U$. Motivation of Pawlak is to characterize the set $U$ with respect to $R$. To this end we will need the basic concepts of rough set theory has given in the next section.

The paper is organised as follows, some preliminaries about rough sets are reviewed in section 2. Consequently, Rough matrix theory is introduced in section 3. Some decision making methods using Rough Matrices are stated and the result is concluded in section 4 .

\section{Preliminaries and notations of rough sets}

Formal definition of approximation and the boundary region are as follows:

Definition 2.1. [3] Let $U \neq \phi$ be a universe of discourse and $\mathrm{X}$ be a subset of $U$. An equivalence relation, $R$, classifies $U$ into a set of subsets $U \backslash R=\left\{X_{1}, X_{2}, \ldots, X_{n}\right\}$ in which the following conditions are satisfied:

1. $X_{i} \subseteq U, X_{i} \neq \phi$ For any i.

2. $X_{i} \cap X_{j} \neq \phi$ For any $\mathrm{i}, \mathrm{j}$.

3. $\bigcup_{R \in P} I N D(R)$

Definition 2.2. [1] Any subset $X_{i}$, which called a category, class or granule, represents an equivalence class of $\mathrm{R}$. A category in $\mathrm{R}$ containing an object $X \in U$ is denoted by $[x]_{R}$. To a family of equivalence relations $P \subseteq R$, an indiscernibility relation over $\mathrm{P}$ is denoted by $I N D(P)$, and is defined by the equation $I N D(P)=\bigcup_{R \in P} I N D(R)$.

\section{Definition 2.3. [3]}

The set $\mathrm{X}$ can be divided according to the basic sets of $\mathrm{R}$, namely a lower approximation set and upper approximation set. Approximation is used to represent the roughness of the knowledge. Suppose a set $X \subseteq U$ represents a 
vague concept, the $\mathrm{R}$-lower and $\mathrm{R}$-upper approximations of $\mathrm{X}$ are defined by the equations

R-lower approximation of $\mathrm{X}: R_{*}(x)=\bigcup_{x \in U}\{R(x): R(x) \subseteq X\}$

R-upper approximation of X: $R^{*}(x)=\bigcup_{x \in U}\{R(x): R(x) \cap X \neq \phi\}$

R-boundary region of X: $R N_{R}(X)=R^{*}(X)-R_{*}(X)$ Also we can define

- R-positive of X: $P O S_{R}(X)=R_{*}(X)$

- R-negative of X: $N E G_{R}(X)=U-R^{*}(X)$

Rough sets are defined by approximations. So rough set definition also requires advanced mathematical concepts[3].

It is easily seen that approximations are in fact interior and closure operations in topology generated by data. Thus fuzzy set theory and rough set theory require completely different mathematical setting. Rough sets can be also defined employing, instead of approximation, rough membership function [1].

Definition 2.4. [3] Rough Membership function defined by Pawlak [3] is given by

$$
\mu_{X}^{R}: U \rightarrow<0,1>\text {, where } \mu_{X}^{R}(x)=\frac{|X \cap R(X)|}{|R(X)|} .
$$

$|X|$ denotes cardinality of $X$.

The rough membership function can be used to define approximations and the boundary region of a set, as shown below;

$$
\begin{aligned}
& R_{*}(X)=\left\{x \in u: \mu_{X}^{R}(x)=1\right\} \\
& R^{*}(X)=\left\{x \in u: 0<\mu_{X}^{R}(x)>0\right\} \\
& R N_{R}(X)=\left\{x \in u: 0<\mu_{X}^{R}(x)<1\right\}
\end{aligned}
$$

\section{Definition 2.5. [3]}

1. Set $\mathrm{X}$ is rough with respect to $\mathrm{R}$ if $R_{*}(X) \neq R^{*}(X)$.

2. Set $\mathrm{X}$ is rough with respect to $\mathrm{R}$ if for some $\mathrm{x}, 0<\mu_{X}^{R}(x)<1$.

Definition 2.6. [2], [4], [5]

An information system is quadruple $I S=(U, A, V, f)$ where $\mathrm{U}$ is a nonempty finite set of objects; A is a non-empty finite set off attributes; $\mathrm{V}$ is the union of domains, $V=\bigcup_{a \in A} V_{a}$, where $V_{a}$ is the value set of attribute a, called the 
domain of a; $f: U \times A \rightarrow V$ is an information function which assigns particular values from domains of attributes to objects such as $\forall a \in A, x \in U, f(a, x) \in V_{a}$ where $f(a, x)$ denotes the value of attribute a for object $\mathrm{x}$.

\section{The Theory of Rough Matrix}

Definition 3.1. An information system is a pair $S=(U, A)$, where $U$ is a non-empty finite set called the universe and $\mathrm{A}$ is a non-empty finite set of attributes, i.e., $a: U \rightarrow V_{a}$ for $a \in A$, where $V_{a}$ is called the domain of a. Elements of $U$ are called objects.

Definition 3.2. An approximation space is a pair $S=(U, R)$, where $U$ is a nonempty finite set called the universe and $R$ is an equivalence relation defined on $U$.

Definition 3.3. A decision table is a special case of information systems, $S=(U, A \cup\{d\})$, where attributes in $A$ are called condition attributes and $d$ is a designated attribute called the decision attribute.

Example 3.4. Example for Approximations:

\begin{tabular}{|c|c|c|c|c|c|c|}
\hline $\mathrm{U}$ & $\mathrm{a}$ & $\mathrm{b}$ & $\mathrm{c}$ & $\mathrm{e}$ & $\mathrm{f}$ & $\mathrm{d}$ \\
\hline 1 & 0 & 0 & 1 & 0 & 0 & 1 \\
\hline 2 & 1 & 1 & 1 & 0 & 0 & 1 \\
\hline 3 & 0 & 1 & 0 & 0 & 0 & 2 \\
\hline 4 & 1 & 0 & 0 & 0 & 1 & 1 \\
\hline 5 & 0 & 1 & 0 & 0 & 0 & 2 \\
\hline 6 & 1 & 0 & 0 & 0 & 1 & 3 \\
\hline 7 & 0 & 0 & 0 & 1 & 1 & 3 \\
\hline
\end{tabular}

\begin{tabular}{|c|c|c|c|c|c|c|}
\hline $\mathrm{U}$ & $\mathrm{a}$ & $\mathrm{b}$ & $\mathrm{c}$ & $\mathrm{e}$ & $\mathrm{f}$ & $\mathrm{d}$ \\
\hline 8 & 1 & 1 & 1 & 1 & 1 & 1 \\
\hline 9 & 0 & 0 & 0 & 1 & 1 & 3 \\
\hline 10 & 0 & 0 & 1 & 0 & 0 & 1 \\
\hline 11 & 1 & 1 & 1 & 0 & 0 & 2 \\
\hline 12 & 1 & 1 & 1 & 1 & 1 & 2 \\
\hline 13 & 1 & 1 & 0 & 1 & 1 & 3 \\
\hline 14 & 1 & 1 & 0 & 0 & 1 & 3 \\
\hline
\end{tabular}

Condition Attributes: $\{a, b, c, e, f\}$, Decision Attributes: $\{d\}$,

Equivalence Classes: Elementary Sets.

$[1]=\{1,10\},[2]=\{2,11\},[3]=\{3,5\},[4]=\{7,9\},[7]=\{7,9\},[8]=\{8,12\}$

$C L A S S(d)=\left\{X_{1}, X_{2}, X_{3}\right\}$, where $X_{1}=[1,2,4,8,10], X_{2}=[3,5,11,12]$,

and $X_{3}=[6,7,9,13,14]$.

The Lower Approximations are

$A_{*}\left(X_{1}\right)=[1] \cup[7]=\{1,7,9,10\}$,

$A_{*}\left(X_{2}\right)=[3] \cup[8]=\{3,5,8,12\}$,

$A_{*}\left(X_{3}\right)=[7] \cup[4]=\{4,6,7,9\}$

The Upper Approximations are

$A^{*}\left(X_{1}\right)=A_{*}\left(X_{1}\right) \cup[2] \cup[4] \cup[8]=\{1,2,4,6,7,8,9,10,11,12\}$,

$A^{*}\left(X_{2}\right)=A_{*}\left(X_{2}\right) \cup[2] \cup[8]=\{2,3,5,8,11,12\}$, 
$A^{*}\left(X_{3}\right)=A_{*}\left(X_{3}\right) \cup[2] \cup[4] \cup[7]=\{2,4,6,7,9,11\}$

The Boundary Regions are

$B N_{A}\left(X_{1}\right)=A^{*}\left(X_{1}\right)-A_{*}\left(X_{1}\right)=\{2,4,6,8,11,12\}$

$B N_{A}\left(X_{2}\right)=A^{*}\left(X_{2}\right)-A_{*}\left(X_{2}\right)=\{2,11\}$

$B N_{A}\left(X_{3}\right)=A^{*}\left(X_{3}\right)-A_{*}\left(X_{3}\right)=\{2,11\}$

Definition 3.5. We can define a Rough Matrix $R_{M}=\left[r_{i j}\right]$ of order $m \times n$ as follows.

$R_{M}=\left[r_{i j}\right]=\left(\begin{array}{ccc}r_{11} & r_{12} & \ldots r_{1 n} \\ r_{21} & r_{22} & \ldots r_{2 n} \\ r_{m 1} & r_{m 2} & \ldots r_{m n}\end{array}\right)$ where each $r_{i j} \in \mu_{x}^{R}$

Definition 3.6. The values of Rough Matrix is defined from approximations as follows.

$$
R_{M}= \begin{cases}1 ; & \text { if } x \in R_{*}(X) \\ (0,1) ; & \text { if } x \in R N_{R}(X) \\ 0 ; & \text { if } x \in U / R_{*}(X)\end{cases}
$$

Definition 3.7. For all $x, y \in[0,1]$, the following operators are defined i) $x \vee y=\max [x, y]$, ii) $x \wedge y=\min [x, y]$

$$
\text { iii) } x / y= \begin{cases}x ; & \text { if } x>y \\ |x| ; & \text { if } x<y \\ 0 ; & \text { if } x=y\end{cases}
$$

iv) $x \oplus y=x+y-x y, \quad v) x \otimes y=x y$

Definition 3.8. $\operatorname{Let}(U, A)$ be a rough set over any approximations, consider subset of $U X A$ is uniquely defined by $R_{A}=\left\{(u, b) ;\right.$ if $u \in R_{A}(b), b \in$ $A\}$ which is called as indiscernibility relation form $(U, A)$. The $R_{A}$ is a function $\phi R_{A}: U X A \rightarrow[0,1]$ defined by

$$
\phi R_{A}= \begin{cases}1 ; & \text { if } x \in R_{*}(X) \\ (0,1) ; & \text { if } x \in R N_{R}(X) \\ 0 ; & \text { if } x \in U / R_{*}(X)\end{cases}
$$

then $R_{M}=\phi R_{A}\left(u_{i}, a_{j}\right)=\left[r_{i j}\right]=\left(\begin{array}{cccc}r_{11} & r_{12} & \ldots r_{1 n} \\ r_{21} & r_{22} & \ldots r_{2 n} \\ r_{31} & r_{32} & \ldots r_{3 n} \\ r_{m 1} & r_{m 2} & \ldots r_{m n}\end{array}\right)$ is called a $m X n$ rough matrix over approximation. The set of all $m \times n$ rough matrices over approximations space will be denoted by $R M_{m \times n}$ 
Definition 3.9. Let $\left[r_{i j} \in R M_{m \times n}\right.$ then $\left[r_{i j}\right]$ is called

i) A Zero Rough Matrix, denoted by [0], if $\left[r_{i j}\right]=0$ for all $x \in U / R_{*}(X)$ ii)A Universal Rough Matrix, denoted by [1],if $\left[r_{i j}\right]=1$ for all $x \in R_{*}(X)$ iii)A Intermediate Rough Matrix,denoted by [0.5],if $\left[r_{i j}\right]=(0,1)$ for all $x \in$ $R N_{R}(X)$

Definition 3.10. Let $A$ and $B$ be two rough matrices or order $m x n$, then we can define the following operations

$$
\begin{aligned}
& \text { i) } \left.\left.A \cup B=\operatorname{Max}\left[A, B^{T}\right] \text {, ii }\right) A \cap B=\operatorname{Min}\left[A, B^{T}\right], \text { iii }\right) A / B=\operatorname{Min}\left[A, \neg B^{T}\right] \\
& \left.\left.\quad \text { iv) } A \oplus B=\left(A \vee B^{T}\right) \vee\left(A \wedge B^{T}\right), \text { v }\right) A \otimes B=\left(A \vee B^{T}\right) \wedge\left(A \wedge B^{T}\right), \text { vi }\right) A \neg B \\
& =\operatorname{Min}\left[\neg A, \neg B^{T}\right]
\end{aligned}
$$

Example 3.11. Let $A$ and $B$ be two rough matrices with entries

$$
A=\left(\begin{array}{cccc}
0 & 1 & 0.5 & 0 \\
1 & 1 & 1 & 0.5 \\
0 & 0 & 0 & 0 \\
1 & 0.5 & 1 & 1
\end{array}\right)
$$

and

$$
B=\left(\begin{array}{cccc}
0 & 0 & 0 & 0 \\
1 & 1 & 1 & 1 \\
0.5 & 0.5 & 0.5 & 0.5 \\
1 & 0 & 0 & 0.5
\end{array}\right)
$$

Then we can find the following rough matrix using definition 3.9.

$$
\begin{gathered}
\neg A=\left(\begin{array}{cccc}
1 & 0 & 0.5 & 1 \\
0 & 0 & 0 & 0.5 \\
1 & 1 & 1 & 1 \\
0 & 0.5 & 0 & 0
\end{array}\right), \quad \neg B=\left(\begin{array}{ccccc}
1 & 1 & 1 & 1 \\
0 & 0 & 0 & 0 \\
0.5 & 0.5 & 0.5 & 0.5 \\
0 & 1 & 1 & 0.5
\end{array}\right), \\
\neg B^{T}=\left(\begin{array}{cccc}
1 & 0 & 0.5 & 0 \\
1 & 0 & 0.5 & 1 \\
1 & 0 & 0.5 & 1 \\
1 & 0 & 0.5 & 0.5
\end{array}\right), \\
A \cup B=\operatorname{Max}\left[A, B^{T}\right]=\left(\begin{array}{cccc}
0 & 1 & 0.5 & 1 \\
1 & 1 & 1 & 0.5 \\
0 & 1 & 0.5 & 0 \\
1 & 1 & 1 & 1
\end{array}\right)
\end{gathered}
$$




$$
\begin{gathered}
A \cap B=\operatorname{Min}\left[A, B^{T}\right]=\left(\begin{array}{cccc}
0 & 1 & 0.5 & 0 \\
0 & 1 & 0.5 & 0 \\
0 & 0 & 0 & 0 \\
0 & 0.5 & 0.5 & 0.5
\end{array}\right), \\
A / B=\operatorname{Min}\left[A, \neg B^{T}\right]=\left(\begin{array}{cccc}
0 & 1 & 0.5 & 0 \\
0 & 0 & 0 & 0.5 \\
0 & 0 & 0 & 0 \\
0 & 0.5 & 1 & 0.5
\end{array}\right), \\
A \oplus B=\left(A \vee B^{T}\right) \vee\left(A \wedge B^{T}\right)=\left(\begin{array}{cccc}
0 & 0 & 0 & 0 \\
1 & 1 & 1 & 0.5 \\
0 & 0 & 0 & 0 \\
1 & 0 & 0 & 0.5
\end{array}\right), \\
A \otimes B=\left(A \vee B^{T}\right) \wedge\left(A \wedge B^{T}\right)=\left(\begin{array}{cccc}
0 & 0 & 0 & 0 \\
1 & 1 & 1 & 0.5 \\
0 & 0 & 0 & 0 \\
1 & 0 & 0 & 0.5
\end{array}\right), \\
A \neg B=\operatorname{Min}\left[\neg A, \neg B^{T}\right]=\left(\begin{array}{cccc}
1 & 0 & 0.5 & 0 \\
0 & 0 & 0 & 0.5 \\
1 & 0 & 0 & 1 \\
0 & 0 & 0 & 0
\end{array}\right)
\end{gathered}
$$

\section{Products of Rough Matrices and its Decision Making}

In this section we are going to define four special products of rough matrices to construct rough decision making methods.

Definition 4.1. Let $\left[a_{i j}\right]$ and $\left[b_{i j}\right]$ be two rough matrices then AndProduct is defined by $R M_{m \times n} \times R M_{m \times n} \rightarrow R M_{m \times n},\left[a_{i j}\right] \wedge\left[b_{i j}\right]=\left[r_{i j}\right]$, where $r_{i j}=\operatorname{Min}\left[a_{i j}, b_{i j}^{T}\right]$.

Definition 4.2. Let $\left[a_{i j}\right]$ and $\left[b_{i j}\right]$ be two rough matrices then Or-Product is defined by $R M_{m \times n} X R M_{m \times n} \rightarrow R M_{m \times n},\left[a_{i j}\right] \vee\left[b_{i j}\right]=\left[r_{i j}\right]$, where $r_{i j}=\operatorname{Max}\left[a_{i j}, b_{i j}^{T}\right]$.

Definition 4.3. Let $\left[a_{i j}\right]$ and $\left[b_{i j}\right]$ be two rough matrices then And-NotProduct is defined by $R M_{m \times n} \times R M_{m \times n} \rightarrow R M_{m \times n},\left[a_{i j}\right] \neg \wedge\left[b_{i j}\right]=\left[r_{i j}\right]$, where $r_{i j}=\operatorname{Min}\left[a_{i j}, 1-b_{i j}^{T}\right]$. 
Definition 4.4. Let $\left[a_{i j}\right]$ and $\left[b_{i j}\right]$ be two rough matrices then Or-NotProduct is defined by $R M_{m x n} X R M_{m x n} \rightarrow R M_{m \times n},\left[a_{i j}\right] \neg \vee\left[b_{i j}\right]=\left[r_{i j}\right]$, where $r_{i j}=\operatorname{Max}\left[a_{i j}, 1-b_{i j}^{T}\right]$.

Working rule to construct RMmDM (Rough Max-min Decision Making) method by the following Algorithm.

1. Choose feasible subsets of the set of attributes.

2. Construct the rough matrix for each set of attributes.

3. Find the convenient product of the rough matrices.

4. Find a Max-min decision rough matrix.

5. Find an optimum set of U.

In similar manner, we can define rough min-Max, rough min-min and rough Max-Max decision making methods.

Example 4.5. Problem Description: Suppose that two friends want to buy a car from $U$, which may be characterized by set of attributes. If each friends has to consider their own set of attributes, then we select a car on the basis of the sets of parters parameters by using $R M m D M$ as follows.

Assume that, set of different cars $U=\left\{c_{1}, c_{2}, c_{3}, c_{4}\right\}$ which is characterized by $A=\left\{x_{1}, x_{2}, x_{3}, x_{4}\right\}$ for $i=\{1,2,3,4\}$ the attributes $x_{i}$ stands for "costly", "mileage", "maintanence free", "cheap", respectively. Let $P_{1}=\left\{x_{1}, x_{3}, x_{4}\right\}$ and $P_{2}=\left\{x_{2}, x_{3}, x_{4}\right\}$ then

$$
R M_{P_{1}}=\left(\begin{array}{cccc}
1 & 0 & 0 & 1 \\
0 & 0 & 0.5 & 1 \\
1 & 0 & 1 & 1 \\
0.5 & 0 & 1 & 0.5
\end{array}\right) \text { and } R M_{P_{2}}=\left(\begin{array}{cccc}
0 & 1 & 0.5 & 1 \\
0 & 1 & 0 & 1 \\
0 & 1 & 1 & 1 \\
0 & 1 & 1 & 0
\end{array}\right)
$$

then

$$
R M_{P_{2}}^{T}=\left(\begin{array}{cccc}
0 & 0 & 0 & 0 \\
1 & 1 & 1 & 1 \\
0.5 & 0 & 1 & 1 \\
1 & 1 & 1 & 0
\end{array}\right) R M_{P_{1}} \wedge R M_{P_{2}}^{T}=\left(\begin{array}{cccc}
0 & 0 & 0 & 0 \\
0 & 0 & 0.5 & 1 \\
0.5 & 0 & 1 & 1 \\
0.5 & 0 & 1 & 0
\end{array}\right)
$$

Now we need to write row-wise maximum as row vector and then pick minimum value on that row vector. That is our required decision. Maximum row vector $=\left(\begin{array}{llll}0 & 1 & 1 & 1\end{array}\right)$ So the decision is car 1 is satisfies both persons expectations. similarly we can define for the remaining cases also. 


\section{References}

[1] Frege and A.Nakamura: conflict logic with degrees, Rough Fuzzy Hybridization - A new trend in decision-making, (S.K.Pal, A.Skowron,eds,),Springer 1999,136-150

[2] Li Xiang-wei, Qi Yian-fang, A data preprocessing algorithm for classification model based on rough sets. Int.Jl.of guangzi teachers education university 2006, vol 23, no.4 pp 87-95.

[3] Z.Pawlak Rough sets Int.J.Inf.Comput.Sci. 11(5)(1982) 341-356.

[4] Yiyu yao, Notes on rough set approximations and associated measures, Journal of zhejiang ocean university (natural science), 29(5)(2010) 399410 .

[5] Yiyu yao and Zhao, Attribute reduction in decision-theoretic rough set models, Information Sciences, 178(17)(2008), 3356-3373. 
